\title{
What is Causing the Deficit of High-Energy Solar Particles in Solar Cycle 24?
}

\author{
R.A. Mewaldt ${ }^{1 \mathrm{a}}$, G. $\mathbf{L i}^{\mathrm{b}}$, J. Hu${ }^{\mathrm{b}}$, and C.M.S. Cohen ${ }^{\mathrm{a}}$ \\ ${ }^{a}$ California Institute of Technology, Pasadena, CA, USA \\ ${ }^{b}$ University of Alabama, Huntsville AL, USA \\ E-mail: RMewaldtecal tech.edu
}

The number of large Solar Energetic Particle (SEP) events in solar cycle 24 is reduced by a factor of about 2 compared to cycle 23 . In the first 8 years of this cycle there have been only 38 "GOES" proton events compared to 79 at this point of cycle 23 . What is less well known is that the fluence of protons and heavier ions is reduced by even greater factors (by 6 times for greater than $10 \mathrm{MeV}$ protons, and by 9 times for greater than $100 \mathrm{MeV}$ protons). Indeed the spectral breaks for $\mathrm{H}, \mathrm{O}$, and $\mathrm{Fe}$ are all occurring about 3 times lower in energy/nucleon in cycle 24. This talk will investigate the reduced acceleration efficiency in cycle 24 by simulating SEP acceleration using the Particle Acceleration and Transport in the Heliosphere (PATH) model, and an improved version known as iPATH, which simulates SEP acceleration at a CME-driven shock in two dimensions. Specifically, we will investigate how SEP fluences and energy spectra depend on variables that include the interplanetary magnetic field strength and turbulence level, and the density and spectrum of suprathermal seed particles.

\footnotetext{
${ }^{1}$ Speaker
} 


\section{Introduction}

Although solar cycle 24 (SC24) started out strong, with a number of sizeable SEP events in 2011 and early 2012, it soon became clear that it would not rival the output of cycles 22 and 23. Figure 1 plots the integrated fluence of $>10 \mathrm{MeV}$ protons versus day of the cycle. Note that cycle 24 led cycle 23 until mid 2012, producing $>50 \%$ of the integrated output to date during the first 3.5 years of the cycle, now in its 9th year. There has only been a single GOES-class event in the last 1.5 years, and it barely exceeded the threshold. The cycle $24>10 \mathrm{MeV}$ fluence is a factor of $\sim 3.9$ to 5.8 below that in cycles 22 and 23, respectively, and a factor of $\sim 6.5$ to 9.1 lower in $>100 \mathrm{MeV}$ protons.

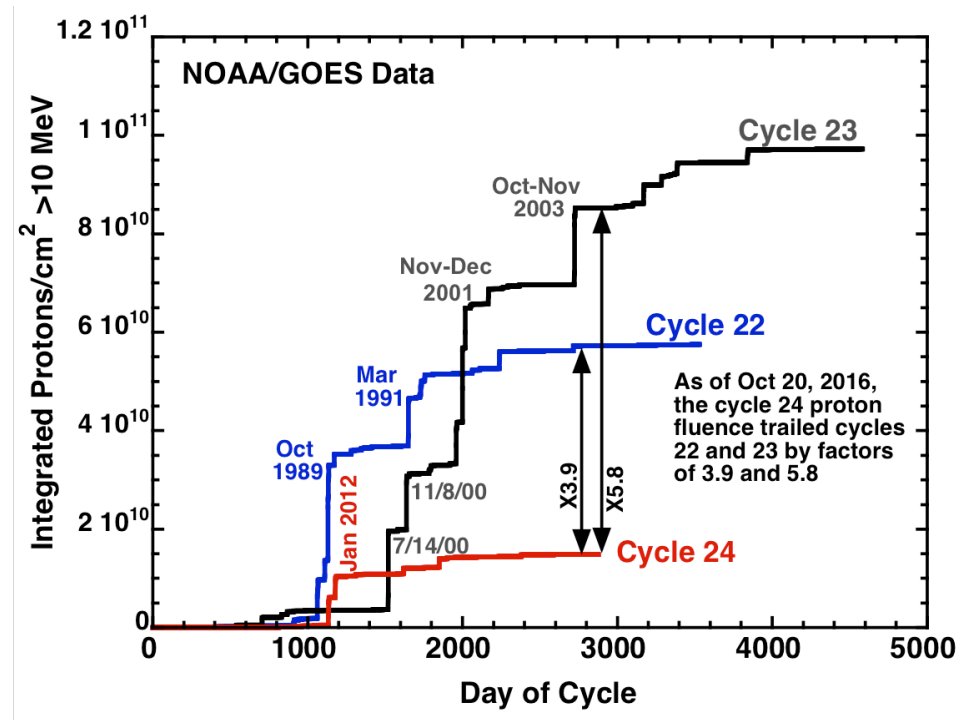

Figure 1: Integrated fluence of $>10 \mathrm{MeV}$ protons versus the day of the cycle for solar cycles 22, 23, and 24 .

In addition, the location of breaks in the fluence spectra of $\mathrm{H}, \mathrm{O}$, and $\mathrm{Fe}$ is reduced by a factor of $\sim 3$ in cycle 24 (Figure 2), and the number densities of sub-MeV seed particles for these species are reduced by factors of $\sim 3$ to $\sim 8$ (see Figure 2). An additional significant difference is that the mean interplanetary magnetic field measured by ACE is $\sim 24 \%$ weaker than in SC23.
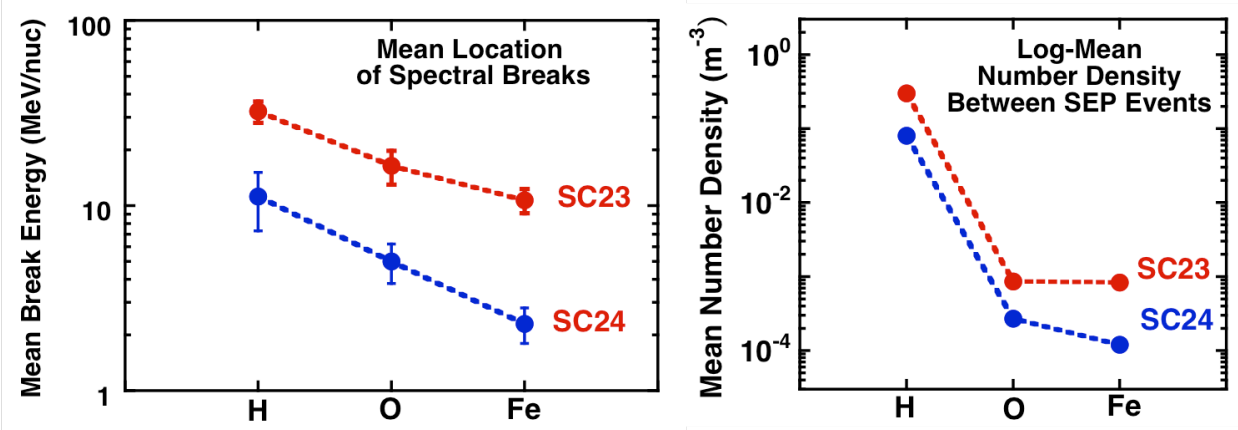

Figure 2: (Left) The mean location of breaks in the H, O, and Fe spectrta averaged over the 10 largest SEP events of Cycles 23 and 24 are shown. (Right) The logarithmic mean of the suprathermal densities of $\sim 0.1$ to $\sim 1 \mathrm{MeV}$ protons measured by ACE/ULEIS in between large SEP events are lower in SC24 by factors of 3 to 8 . 
Table 1 compares other aspects of cycles 23 and 24 . Since there has been only 1 small "GOES-class" SEP event since 2015 we have not brought it completely up to date. There have clearly been fewer X-class flares in cycle 24, but most high-energy SEPs are accelerated by CME driven shocks, not flares. Thus the lower number of fast CMEs appears to account for the factor of $\sim 2$ reduction in the number of $>10 \mathrm{MeV}$ GOES events. However, the reduced number of fast CMEs doesn't account for a factor of 6 to 9 reduction in SEP fluences in cycle 24 . Evidently, fast CMEs are less efficient accelerators in cycle 24 (see also [1]).

\begin{tabular}{|c|c|c|c|c|}
\hline Property & Cycle 23 & Cycle 24 & $\begin{array}{l}\text { Ratio } \\
24 / 23\end{array}$ & Comments/References \\
\hline X-Flares & 88 & 28 & 0.32 & Solar Soft Catalog \\
\hline $\begin{array}{c}\text { CMEs } \\
>1000 \mathrm{~km} / \mathrm{s}\end{array}$ & 296 & 168 & 0.57 & SOHO CME Catalog \\
\hline GOES SEPS & 78 & 39 & 0.50 & NOAA GOES Data \\
\hline $\begin{array}{c}\text { Number of } \\
\text { GLEs }\end{array}$ & 11 & $1-2$ & $0.1-0.2$ & 5/1/12; 1/6/14?, \\
\hline$<\mathrm{B}>\mathrm{IMF}$ & 6.8 & 5.2 & 0.76 & ACE/MAG@L1 \\
\hline $\begin{array}{l}\text { Suprathermal } \\
\text { Seed Density }\end{array}$ & $0.3 / \mathrm{m}^{\wedge} 3$ & $0.080 / \mathrm{m}^{\wedge} 3$ & 0.27 & ACE/ULEIS \\
\hline $\begin{array}{l}\text { H Fluence } \\
>10 \mathrm{MeV}\end{array}$ & $6.3 \times 10^{8}$ & $9.7 \times 10^{7}$ & 0.15 & NOAA /GOES \\
\hline
\end{tabular}

In this paper we consider two factors that may combine to produce the lower SEP output, the weaker interplanetary magnetic field (IMF) and a significant reduction in the mean density of suprathermal seed particles [1]. We also present some initial modeling results that attempt to test the effect of these factors. For other investigations of these topics, see Gopalswamy et al. [2], and Giacalone [3].

\section{Shock Acceleration Considerations}

The long solar minimum preceding SC24 had two solar rotations in 2009 with the weakest IMF of the space era [4]. The SC24 field has remained weaker than SC23 by an average of $\sim 24 \%$ [1]. In a 2006 paper Zank et al. [5] considered factors affecting the maximum proton energy for CME shock acceleration, including CME velocity, shock compression ratio, seed particle injection rate, and a combination of the IMF strength and turbulence level that they represented as $\left(\delta \mathrm{B}^{5} / \mathrm{B}^{6}\right)$. We tested this combination of factors, and while $\mathrm{B}$ is reduced in $\mathrm{SC} 24$, we found no evidence for a reduction in $\delta \mathrm{B} / \mathrm{B}$ from cycle 23 to cycle 24 [1]. However, $\mathrm{B}$ is important because it limits the acceleration rate (since $\mathrm{dE} / \mathrm{dt}$ is proportional to $\mathrm{B}$, see, e.g., [6]).

The density of suprathermal seed particles in the acceleration region is also a key factor because they provide most of the ions accelerated by CME-driven shocks [7,8]. It is not generally appreciated, but in standard diffusive shock acceleration (DSA) theory (e.g., Lee [9]) it is the injection rate of suprathermal protons that controls the ion acceleration rate because accelerated ions escaping upstream from the shock excite and amplify Alfven waves that trap 
particles near the shock more efficiently, thereby increasing the maximum energy of escaping particles in a non-linear fashion (e.g., [10,1]).

The PATH model was first introduced by Zank et al. [11]. The core of the original model consists of two parts: (1) a one-dimensional hydrodynamic ZEUS code that simulates background solar wind (assumed spherically symmetric) and a propagating CME-driven shock; (2) an onion shell module where injected particles are accelerated by diffusive shockacceleration (DSA) and diffuse between shells within the shock complex (including the shock downstream region and diffusive region ahead of the shock). Particles that diffuse far enough upstream of the shock are assumed to escape, at which point they propagate ballistically into the interplanetary medium.

The PATH model has been improved over time. Rice et al. [12] considered shocks of arbitrary strength, providing improved estimates of maximum particle energies. Li et al. [13] extended PATH by modeling the transport of SEPs escaping the shock using a direct Boltzmann-Vlasov description. By adopting a mean-free-path dependent on both particle energy and heliocentric distance, they obtained more realistic SEP time-intensity profiles and spectra at $1 \mathrm{AU}$. Li et al. [14] further extended the model to include heavy ions. They showed that heavy-ion spectral shapes scale with ionic charge $(\mathrm{Q})$ and mass $(\mathrm{A})$ as $(\mathrm{Q} / \mathrm{A})^{2}$ at parallel shocks. Li et al. [15] showed this scaling depends on shock obliquity. [e.g. for highly perpendicular shocks the $(\mathrm{Q} / \mathrm{A})$ scaling can be as shallow as $\left.(\mathrm{Q} / \mathrm{A})^{1 / 9}\right]$.

Our first tests of the non-linear nature of shock acceleration discussed above were done using the PATH model. Figure 3 shows two spectra generated by PATH for a quasiparallel shock driven by a $2200 \mathrm{~km} / \mathrm{s}$ CME. The left panel assumes an injection rate given by $\varepsilon \mathrm{N}=5 \mathrm{e}-5$, where $\mathrm{N}$ is the upstream density of seed protons. The injection rate is twice as great in the right panel. Note that the spectral break on the right is $57 \%$ greater in energy than that on the left and the maximum energy is more than twice as high. We suggest that the much lower seed particle density in SC24 played a key role in limiting the maximum energy and fluence of SEP events in SC24.
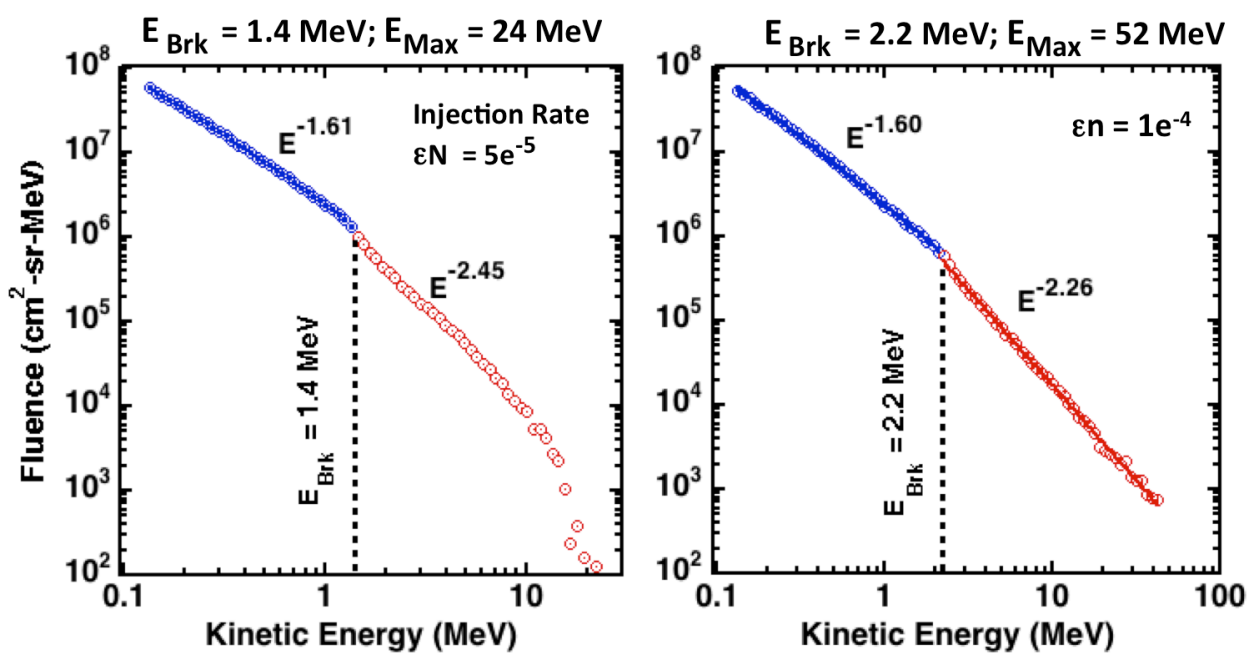

Figure 3: Simulated spectra from the PATH code for protons accelerated at a shock driven by a $2200 \mathrm{~km} / \mathrm{s}$ CME. The suprathermal seed population had a spectrum given by $\mathrm{f}(\mathrm{E})=$ $\mathrm{f}\left(\mathrm{E}_{\mathrm{o}}\right)\left(\mathrm{E} / \mathrm{E}_{\mathrm{o}}\right)^{-3.5}$. The suprathermal injection rate was taken to be $\mathrm{eN}=5 \mathrm{e}^{-5}$ in the left panel and twice as great for the right panel. Note that on the right the break occurs $\sim 50 \%$ higher in energy, while the maximum energy is $\sim 2$ times as great. 


\section{The iPATH Model}

The 1-D PATH model was recently extended to a time-dependent 2-D iPATH model [16]. The major improvement is that modeling particle acceleration and transport in 2-D yields the longitudinal dependence of physical quantities of interest (e.g. time-intensity profiles, particle spectra, pitch-angle distributions). The iPATH model generates a 2D CME-driven shock with a more realistic geometry in the MHD module. Particle acceleration at the shock depends on both parallel and perpendicular diffusion coefficients and is therefore shock-obliquity dependent. Once accelerated, particles diffuse and convect in the shock complex, using a refined 2-D shell model. When particles escape the shock, they propagate along and across the IMF. The propagation is modeled using a focused transport equation with the addition of perpendicular diffusion. The transport equation is solved using a backward stochastic differential equation method where adiabatic cooling, focusing, pitch-angle scattering, and cross-field diffusion effects are all included.

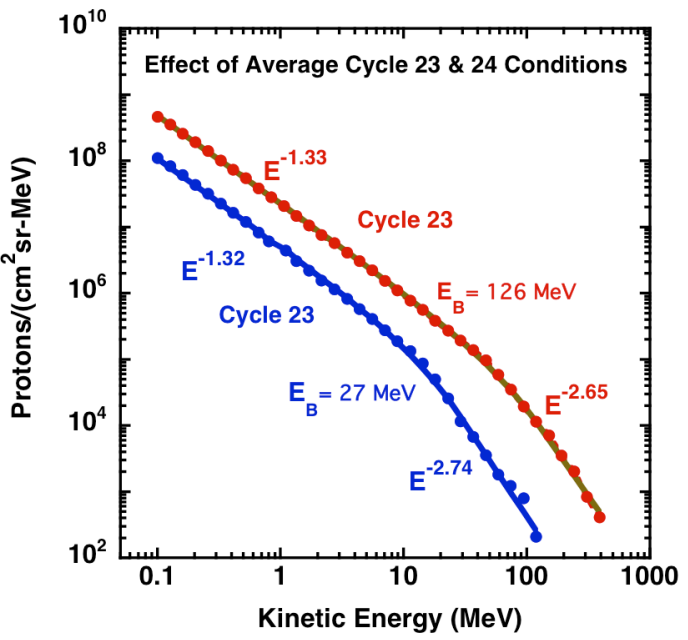

Figure 4: A comparison of fluence spectra generated by iPATH simulations that were tuned to the mean solar wind and seed-particle properties of SC23 and SC24. The circles show energy bins output by iPATH. The solid colored curves are fits to the double-power-law Band function [17].
As an example of how the reduced IMF strength and lower seedparticle densities could have affected SEP fluences in SC24 we used the iPATH code to simulate events characterized by the mean interplanetary conditions of the two cycles. Thus we normalize the IMF strengths to the mean 1-AU values of $6.8 \mathrm{nT}$ in $\mathrm{SC} 23$ and $5.2 \mathrm{nT}$ in $\mathrm{SC} 24$ (Table 1). We also use mean solar wind velocities of $426 \mathrm{~km} / \mathrm{s}$ in SC23 and $401 \mathrm{~km} / \mathrm{s}$ in SC24. To simulate differences in the seed-particle densities we used a seed-particle injection efficiency that is 3.6 times lower in SC24. A CME velocity of $1100 \mathrm{~km} / \mathrm{s}$ was assumed for all runs.

The resulting fluence spectra (see Figure 4) have very similar spectral shapes, but the $>10$ $\mathrm{MeV}$ fluence for SC23 conditions is a factor of $\sim 7$ greater than for SC24 conditions, increasing to a factor of $\sim 33$ greater at $30 \mathrm{MeV}$ (somewhat more than the observed cycle 23 to cycle 24 fluence ratios, see Figure 1). In order to determine the relative impact of the stronger interplanetary magnetic field and greater seed particle injection rate in solar cycle 23 , we did a run with cycle 23 conditions, but lowered the IMF strength to the cycle 24 level of $5.2 \mathrm{nT}$. A second run was done with a $6.8 \mathrm{nT}$ IMF but a seed-particle injection rate equal to that in SC24. Table 2 summarizes the results of these runs. 


\begin{tabular}{|c|c|c|c|c|c|}
\hline \multicolumn{6}{|c|}{ Table 2: Modeled SEP Properties vs. IMF Conditions } \\
\hline Run & $\begin{array}{c}\text { 1-AU IMF } \\
\text { Strength (nT) }\end{array}$ & $\begin{array}{c}\text { Proton Injection } \\
\text { Efficiency }\end{array}$ & $\begin{array}{c}\text { Emax } \\
\text { (MeV) } \\
\text { (averaged) }\end{array}$ & $\begin{array}{c}\text { Break } \\
\text { Energy } \\
\text { (MeV) }\end{array}$ & $\begin{array}{c}\mathbf{> 1 0} \text { MeV } \\
\text { Fluence } \\
\text { (cm } \mathbf{s r})\end{array}$ \\
\hline 1 & 6.8 & $3.6 \%$ & 234 & 126 & $1.2 \times 10^{7}$ \\
\hline 2 & 5.4 & $1 \%$ & 186 & 27 & $9.6 \times 10^{5}$ \\
\hline 3 & 5.4 & $3.6 \%$ & 186 & 23 & $1.1 \times 10^{6}$ \\
\hline 4 & 6.8 & $1 \%$ & 227 & 25 & $1.1 \times 10^{6}$ \\
\hline
\end{tabular}

As might be expected, the cycle-23 conditions in Run 1 have greater maximum energies (averaged over the shock), larger spectral break energies, and greater $>10 \mathrm{MeV}$ fluences than result from the cycle 23 conditions (Run 2). A comparison Run 1 with Run 4 (which had cycle 23 conditions except for a reduced injection efficiency, suggests that the large fluences in Run 1 are due mainly to the higher proton injection efficiency associated with a higher density of seed particles. A comparison of Run 1 and Run 3 suggests that the stronger IMF in Run 1 was mainly responsible for the much higher break energy and greater Emax of Run 1. These preliminary conclusions need to be tested with runs carried out over a greater range of initial conditions. These spectra can also be fit by an Ellison-Ramaty spectrum (power-law multiplied by an exponential [18]).

\section{Summary}

There are a number of reasons why solar energetic particle activity has been greatly reduced during solar cycle 24 . Certainly the factor of $\sim 2$ reduction in large solar events and fast CMEs has played an important role. A secondary effect of the reduced solar activity is generally weaker fields in the corona and interplanetary medium, as well as a reduced level of suprathermal seed particles for the shocks to accelerate when they do occur. We suggest that another key factor is the non-linear nature of CME-shock acceleration. It has been claimed that the largest SEP events occur when a fast CME follows on the heels of an earlier one from the same active region $[19,20]$. Although there may also be other factors at play, the seed particles generated by the first CME-shock provide a natural means of improving the acceleration efficiency at the second shock.

Although we are still learning how to use the iPATH model, in future work we plan to thoroughly explore the role of solar wind, suprathermal ion, CME, and shock properties in determining the intensity of SEP events. We will also model some of the interesting examples of multi-spacecraft events from the past cycle and prepare for the coming era of the Parker Solar Probe and Solar Orbiter missions.

Acknowledgements: Work at Caltech and the University of Alabama/Huntsville was funded by a SHINE Collaborative Research grant under NSF Grant 1622487. In addition, Caltech was funded in part by NASA grants NNZ13AH66G and NNX15AG09G, and UA 
Huntsville was funded in part by NSF grant AGS-1622391, NASA grants NNX15AJ93G and NNX14AC08G, and by a 2013-2014 IIDR grant at UAH.

\section{References}

[1] R. A. Mewaldt, C. M. S Cohen et al., "Investigating the Causes of Solar-Cycle Variations in Solar Energetic Particle Fluences and Composition", in Proceedings of Science, $34^{\text {th }}$ International Cosmic Ray Conference (2015).

[2] N. Gopalswamy, Hong Xie, et al., "Major Solar Eruptions and High-Energy Particle Events during Solar Cycle 24”, Earth, Planets and Space, 66:104, doi:10.1186/1880-5981-66-104 (2014).

[3] J. Giacalone, "Diffusive Shock Acceleration of High-Energy Spectra of Ground-Level Events during Shock Acceleration of High Energy Charged Particles at Fast Interplanetary Shocks: A Parameter Study: Astrophysical Journal, 799:80, doi:10.1088/0004-637X/799/1/80 (2015).

[4] R. A. Mewaldt, A. J. Davis, et al., "Record Breaking Cosmic Ray Intensities During 2009-2010”, Astrophysical Journal Letters 723, L1, doi:10.1088/2041-8205/723/1 (2010).

[6] T. K. Gaisser, Cosmic Rays and Particle Physics, Cambridge University Press, Cambridge (1993).

[7] G. M. Mason, J.E. Mazur, et al., "3He Enhancements in Large Solar Energetic Particle Events", Astrophysical Journal 525, L133 (1999).

[8] R. A Mewaldt, C. M. S. Cohen, et al., "The Dependence of Solar Energetic Particle Fluences on Suprathermal Seed-Particle Densities", in Space Weather: The Space Radiation Environment, Q. $\mathrm{Hu}$ et al. editors, AIP Conference Proceedings 1500 (2012).

[9] M. A. Lee, "Coupled Hydromagnetic Wave Excitation and Ion Acceleration at Travelling Interplanetary Shocks", Journal of Geophysical Research, 88, 6109 (1983).

[10] M. A. Lee, J. Giacalone, et al, "Shock Acceleration of Ions in the Heliosphere", Space Science Reviews 143, 247, doi:10.1007/s11214-012-9932-y (2012).

[11] G. P. Zank, W. K. M Rice, et al., Particle Acceleration and Coronal shocks: A Theoretical Model, Journal of Geophysical Research 105, 25079 (2000).

[12] W. K. M. Rice, G. P. Zank, et al., "Particle acceleration and coronal mass ejection driven shocks: shocks of arbitrary strength”, Journal of Geophysical Research: 108, 1 (2003).

[13] G. Li "CME-driven shocks, coronal mass ejections (CMEs), diffusive shock acceleration, energetic particles”, Journal of Geophysical Research, 110, 1 (2005).

[14] G. Li, "Acceleration and transport of heavy ions at coronal mass ejection-driven shocks", 2005), Journal of Geophysical Research, 110, A06104, (2005).

[15] G. Li, G. P. Zank, et al., "Shock Geometry and Spectral Breaks in Large SEP Events" Astrophysical Journal, 702, 998 (2009).

[16] J. Hu, G. Li, et al., "Modeling Particle Acceleration and Transport at a 2D CME-Driven Shock", submitted to Journal of Geophysical Research, 2017.

[17] D. Band, J. Matteson, et al., BATSE Observations of Gamma-Ray Burst Spectral-Diversity, Astrophysical Journal 413, 28 (1993). 
[18] D. C. Ellison and R. Ramaty, "Shock Acceleration of Electrons and Ions in Solar Flares", Astrophysical Journal 298, 400 (1985).

[19] N. Gopalswamy, S. Yashiro, et al., "Intensity Variation of Large Solar Energetic Particle Events associated with Coronal Mass Ejections", Journal of Geophysical Research, 109, A12105, doi:10.1029/2004JA010602 (2004).

[20] L. G. Ding, G. Li, et al., 2015, "Seed Population in Large Solar Energetic particle Events and the Twin CME Scenario”, Astrophysical Journal, 812, 171 (2015). 